\title{
LIFELONG LEARNING: A SKILl NeEded Today FOR THE ENGINEERS OF THE FUTURE
}

\author{
Sadegh Babaii Kochekseraii and Libby Osgood \\ School of Sustainable Design Engineering, University of Prince Edward Island \\ sbabaii@upei.ca, eosgood@upei.ca
}

\begin{abstract}
This paper will focus on our efforts to introduce the lifelong learning graduate attribute into the classroom environment required by CEAB for engineering accreditation. ENGN334: Intro to Mechatronics is a third year focus area elective course in the new engineering degree at UPEI. It gave the opportunity to develop a syllabus in which the students were encouraged to proactively participate in developing their own weekly learning goals based on the proposed list of topics. From their weekly submissions and subsequent reflections, we tried to answer if the students were setting realistic goals, assessed against SMART learning goals, and how the balance of the short and long term goals changed over the semester. It is therefore the objective of this paper to examine how effective it could be to promote realistic goal setting through professional skill development $(P S D)$ intervention and proactive selfdirected learning.
\end{abstract}

Keywords: CEAB graduate attributes, lifelong learning, SMART learning goals

\section{INTRODUCTION}

Industry expects engineering graduates to perform their assigned tasks almost immediately out of school. To help graduates meet this expectation, the Canadian Engineering Accreditation Board (CEAB) has developed a set of attributes that all students studying engineering should acquire by end of their degree program. Attributes such as knowledge of engineering, use of tools, problem solving, teamwork and design are actively taught. On the other hand, attributes such as equity, ethics, professionalism and lifelong learning have been treated as implicit learning. For example, development of lifelong learning has always been taken for granted as a result of completing a degree program. The CEAB appears to insist that all attributes are explicitly introduced, developed, applied and assessed in the curriculum.

The University of Prince Edward Island (UPEI), School of Sustainable Design Engineering (UPEI
Engineering) is introducing a vanguard engineering education program concept. It is orientated around project based design, learning and professional practice courses through all four years of study with emphasis on professional skill development (PSD), hands-on content and experiential learning elements. As a result, many new courses have had to be approved and introduced to the curriculum to meet this objective. It has provided the teachers with a green field opportunity to develop new ways of actively introducing concepts such as equity, ethics, professionalism and lifelong learning.

Engineering graduates working in the industry and practicing professional engineers are required to set yearly professional development goals and show evidence of achieving set career objectives. We argue that this practice in the industry is similar to the $\mathrm{CEAB}$ requirement for the lifelong learning skills. With no other engineering course known to us that proactively introduces, applies and then assesses lifelong learning habits and strategies, we experimented with one strategy using focus area elective course ENGN 334: Introduction to Mechatronics. Our intention was to introduce lifelong learning strategy to the students and to differentiate it from their day to day learning necessary to complete the engineering program. It was an opportunity to be able to develop a syllabus that encouraged the students to proactively participate in developing their own weekly learning goals based on the proposed study outline. An intervention was given in form of a PSD on how to write achievable and meaningful learning goals, just before the midterm assessment.

From their weekly submissions and subsequent reflections on their learning achievements at the end of the weekly periods, we hoped to answer:

1. Are students setting realistic goals?

2. How does the balance of short term and long term goals change over the semester?

3. How proactively are students connecting to course material as listed in the syllabus?

4. Do they ask for topics to be covered to ensure their goals are met? 
It is therefore the objective of this paper to examine how effective it could be to promote realistic goal setting and search for learning materials through active learning. This paper concentrates on answering the first two questions above, while the third and fourth questions will be addressed in a future study.

\section{BACKGROUND}

In 2008, the CEAB published a set of new requirements [1] in which included a list of graduate attributes specific to an outcome based accreditation criteria. Section 3.1 of this document lists 12 graduate attributes all students should have acquired upon completion of an accredited engineering program. The last in the list was lifelong learning skills.

Cloutier et.al. [2] published their efforts on mapping CEAB outcome based accreditation to existing CDIO based engineering syllabus [3]. While CDIO was found to be an effective tool for teaching Design, teamwork and even professionalism, it was challenging for introducing attributes such as lifelong learning skills or ethics and equity.

The CEAB defines lifelong learning graduate attribute as "An ability to identify and to address their own educational needs in a changing world, sufficiently to maintain their competence and contribute to the advancement of knowledge" [1]. At UPEI Engineering, the definition was further elaborated to cover stages "Introduced" through "Developed" as follows:

1. Recognize the need for self-learning skills and reflection,

2. Compose reflections evaluating personal work performance,

3. Define personal, interpersonal and professional skills themselves,

4. Identify knowledge gaps and learning needs,

5. Practice improvement of personal, interpersonal and professional skills, and

6. Set personal self-learning goals

Students are taught the learning goal writing process incorporating Bloom's Taxonomy of Educational Objectives [4].

SMART criteria were used to test the effectiveness of the learning objectives as described in "The Power of SMART Goals" by O'Neill et.al. (2006)[5]. The methodology is described further in section three. Tofade et.al. [6] in their study regarding Continued Professional Development $(\mathrm{CDP})$ of $1^{\text {st }}$ year and $2^{\text {nd }}$ year pharmacy students concludes "with focused online or live training, students are capable of incorporating principles of CPD by writing SMART learning objectives."

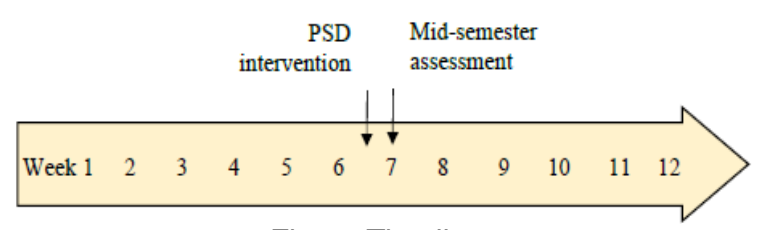

Fig. 1. Timeline.

\section{METHOD}

In the fall 2015 semester, ten students in were introduced to goal-setting. Students completed twelve weekly assignments to set goals for the upcoming week and documented the status of goals from the previous week. There was an intervention between the sixth and seventh submission, where a guest lecturer instructed how to define goals and provided an application of the exercise to industry using a PSD. There was a mid-semester assessment after the seventh assignment where students reflected on the achievement of their goals for the first half of the semester and rated their progress. The timeline can be seen in Figure 1. At the end of the semester, permission was requested from the ten students to use their learning objective for the research-ethics board approved study.

This study is a qualitative analysis of the learning objectives from the twelve assignments and mid-semester assessment. The sample consists of four male third-year engineering students. To answer question one above participants referred to as S1 to S4, were asked to identify and set a number of weekly learning goals. Each learning objective was given a quality score from 0 to 5 , with one point for each of the following SMART [5] criteria:

- Specific: A clearly defined, specific goal

- Measureable: Containing ways to access progress

- Attainable: Can be attained within set time frame using available resources

- Relevant: Being relevant to the targeted topic

- Time bound: Having a specific time frame to complete the goal.

The results were averaged each week for each student and plotted against weeks one to twelve of the Fall term 2015 by two raters.

To answer question two above each learning objective was categorized by intended duration: short-term (one week), mid-term (2-3 weeks), or long-term (4 or more weeks), to document the learning needs and to forecast changes throughout the semester. The goals were assessed as to whether they were task-oriented or learning process oriented. The quality, duration, and outcome were reviewed by two independent reviewers to increase validity and reliability of data review. 


\section{RESULTS AND DISCUSSIONS}

We would like to present the collected data:

- Against SMART criteria to see if students had acquired some lifelong learning skills managing their learning goals prior to starting ENGN 334.

- Investigate if learning goals got any SMARTer after receiving the PSD.

- And, to discuss is it better to promote longer term goals vs weekly goals?

\subsection{Realistic Learning Goals}

Figures 2 and 3 present the results of analysis of weekly learning goals using SMART criteria by the authors as raters 1 and 2 respectively. The effect of the $\mathrm{PSD}$, given on week 6 , is clearly reflected on the results. Class averaged SMART scores given by two raters were plotted for weeks one to twelve and compared with each other shown in Figure 4. Despite agreement between two raters before and immediately after the PSD intervention, their assessments diverged the term progressed after the PSD.

Reviewing the data before and after the PSD, we found the following facts:

1. Before the intervention, goals were written in sporadic form, not following Bloom's Taxonomy.

2. They lacked multiple elements of SMART criteria and therefore both raters concurred in their assessments.

3. After the intervention, students used wording recommended by Bloom's Taxonomy. This reflected as an immediate jump in the SMART assessment by both raters.

4. As weeks progressed after the PSD to the end of the term, students set more goals that were task based to be completed in the week.

Of course for the students, achieving their set tasks implied associated learning. The learnings were implicit, not being explicitly identified by the students as their intended goal. The two raters seemed to differ in their opinion of relevance of the goals based on tasks and not the underlying learnings. Therefore, explaining the observed diverging trend as increasing number of students in the class were setting more of their weekly goals in task based form.

In lieu of the obtained results, we are still uncertain on relevance of tasked based goals with implicit learning versus explicitly identified learning goals. Rater 1 considered practicing setting SMART goals and the associated learning as an important step in developing lifelong learning skills in engineering students. Rater 2, on the other hand, had the opinion that it is through explicitly identified learning goals that student would acquire skills to solve the immediate task plus acquiring an identifiable lifelong learning. In a professional career span, all task based learnings could be considered short term and would not necessarily benefit long term professional development of practicing engineers.

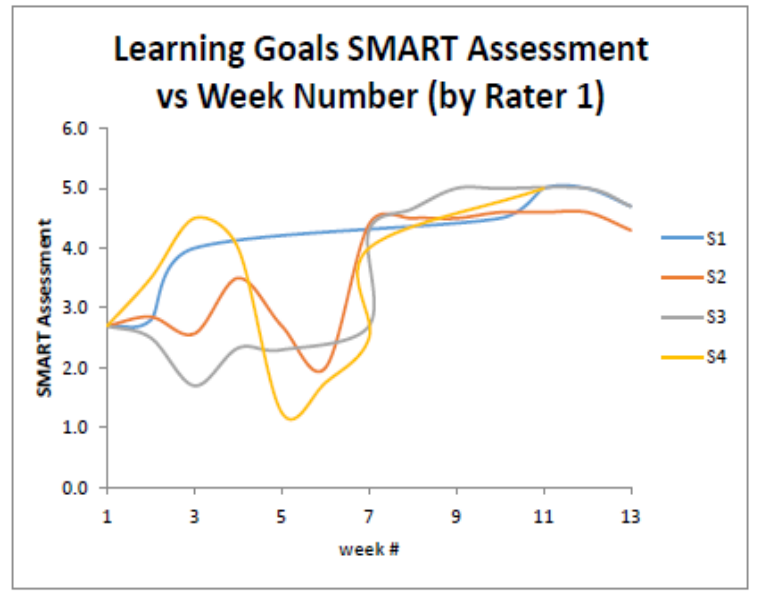

Fig. 2. Assessment of weekly learning objectives using SMART Criteria by rater 1 .

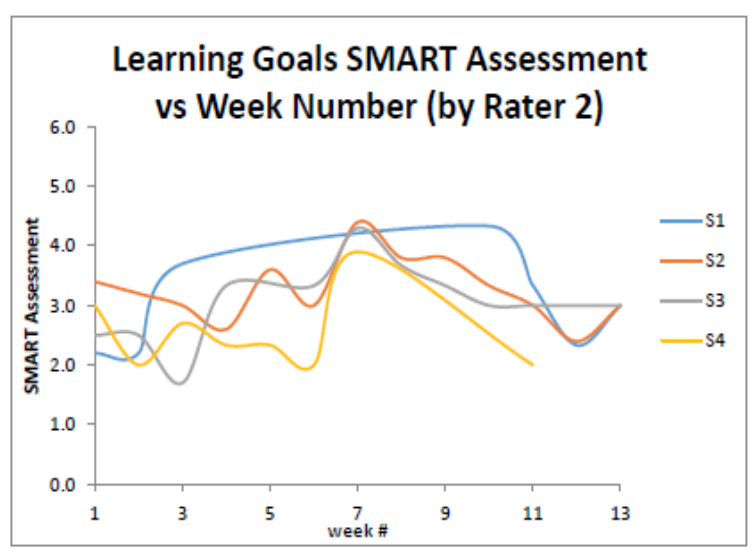

Fig. 3. Assessment of weekly learning objectives using SMART Criteria by rater 2 .

\subsection{Short-Term vs Long-Term Goals}

SMART emphasizes on Time bounded learning goals. SMART assessment requires learning goals to be both time bounded and attainable within the set time frame. The result of our analysis of the available data to identify the ratio between short-term, medium-term and long-term goals is presented in Figure 5. It shows a clear trend by students to set more medium-term and long-term goals as the term progressed. Significant week to week fluctuations are observable but the underlying increase in the percentage of the longer- term goals compared to short-term weekly goals clear. We believe the observed trend is due to an increasing number of students realizing 
the need to set longer term goals to satisfy their learning requirements. Therefore, an increased percentage of longer-term goals were set from the total number set for the week. This trend was continuous from week 1 to the end of the term, and independent of the PSD intervention given in week 6 .

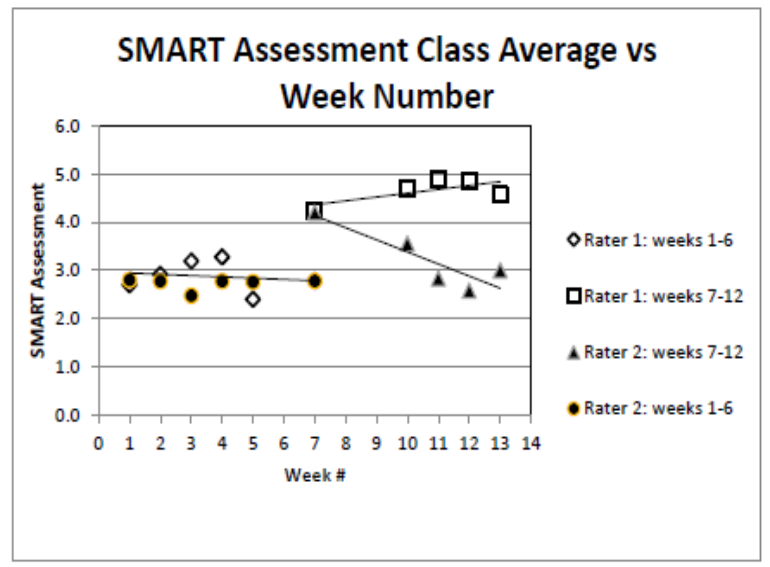

Fig. 4. Class average SMART assessment by raters 1, and 2 , before and after the PSD intervention.

Students were asked to reflect on their weekly learning. This reflection writing was believed to be the other significant factor. We believe it was influential in developing a sense of the time requirement to achieve their learning goals and why they need to carry on learning certain topics over multiple weeks to reach the desired learning outcome. We therefore suggest promoting a balanced approach to setting learning goals in the form of approximately $60 \%$ dedicated to short to medium-term goals and the rest dedicated to long-term learning goals.

\section{CONCLUSIONS AND FUTURE WORK}

The study was a direct result of the initiative by the corresponding author to proactively introduce and apply CEAB graduate attribute lifelong learning in UPEI engineering elective course ENGN 334: Intro to Mechatronics. At the end of the study the following conclusions could be made:

- Lifelong learning and realistic goal setting skills should be explicitly introduced in the engineering curriculum.

- SMART framework for setting learning goals using action words and lexicon suggested by Bloom's Taxonomy are found to be useful tools to be introduced to students early in their engineering curriculum and reinforced regularly.

- A balanced mix of long term and short term learning goals should be suggested to students as an effective approach to manage their lifelong learning to achieve both tasks at hand and long term career objectives.

- Tendency to set task, short term goals should be balanced with long term learning goals serving career objectives not necessary related to tasks in hand.

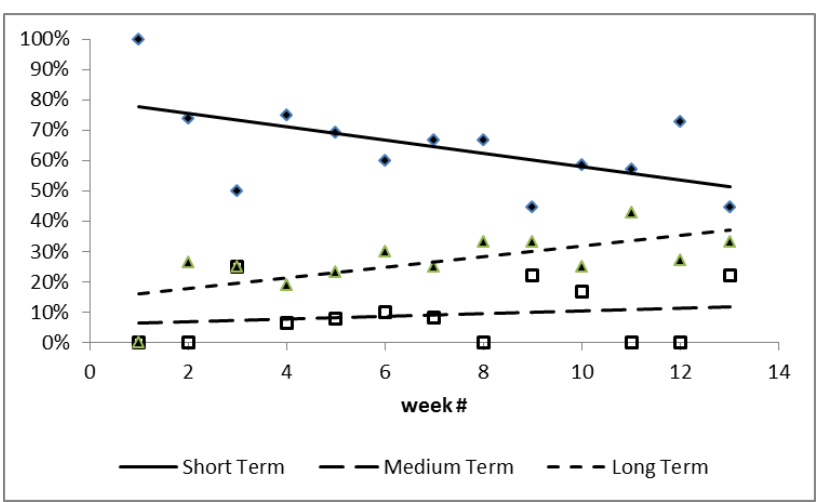

Fig. 5. Long term goal percentages vs short term goals

With these conclusions, we expect to continue the weekly goals setting exercise we started in ENGN334 during Fall term 2015. In Fall term 2016, we expect a larger class population and more collected data to add to what already been gathered. We will try to investigate if the same conclusions are still valid with more available data as well as having opportunity to answer other questions of interest like:

- How proactively are students connecting to course materials listed in the syllabus? (objective 3 )

- Or, how do they ask for topics to be covered to ensure their goals are met? (objective 4)

- And, what would be the effect of introducing the PSD earlier, say two weeks after start of the term.

\section{References}

[1] Canadian Engineering Accreditation Board , 2014 Accreditation Criteria and Procedures / Bureau canadien d'agrément des programmes de génie: Normes et procédures d'agrément. Ottawa, ON: Engineers Canada / Ingénieurs Canada, 2014. 116pp. \{ISSN 1708-8054\}. Available from https://www.engineerscanada.ca/sites/default/files/2014_accr editation_criteria_and_procedures_v06.pdf

[2] Guy Cloutier, Ronald Hugo, and Rick Sellens, "Mapping the relationship between the CDIO syllabus and the 2008 CEAB graduate attributes," in Proc. the 6th International CDIO Conference, (Montreal, QC; 15-18 June 2010), 10 pp., 2011 
[3] Edward Crawley, Johan Malmqvist, Sören Östlund, and Doris Brodeur, Rethinking Engineering Education: The CDIO Approach. New York, NY: Springer, 2010 (2nd ed.), 300 pp. \{ISBN: 978-1441-94260-9\}

[4] Benjamin S. Bloom, M. D. Engelhart, E. J. Furst, W. H. Hill, and David R. Krathwohl, Taxonomy of Educational Objectives: The classification of educational goals. Handbook I: Cognitive domain. New York, NY: David McKay Company, 1956.
[5] Jan O'Niell, Anne Conzemius, The Power of SMART Goals: Using Goals to Improve Student Learning. Bloomington, IN: Solution Tree Press, 2006, 194pp. \{ISBN 978-1932127-87-4\}

[6] Toyin Tofade, Anand Khandoobhai, and Kim Leadon, "Instructional design and assessment: Use of SMART learning objectives to introduce continuing professional development into the pharmacy curriculum, " American Journal of Pharmaceutical Education, vol. 76, no. 4, Article 68, 2012. 